Bulletin d'études orientales

LXIV | 2016

Histoire et anthropologie des odeurs en terre d'Islam à l'époque médiévale - Année 2015

\title{
Entre fragrances et pestilences, étudier les odeurs en terre d'Islam au Moyen Âge
}

Julie Bonnéric

\section{(2) OpenEdition \\ Journals}

Édition électronique

URL : http://journals.openedition.org/beo/4689

DOI : $10.4000 /$ beo.4689

ISSN : 2077-4079

Éditeur

Presses de l'Institut français du Proche-Orient

Édition imprimée

Date de publication : 1 avril 2016

Pagination : 21-42

ISBN : 978-2-35159-800-9

ISSN : 0253-1623

Référence électronique

Julie Bonnéric, "Entre fragrances et pestilences, étudier les odeurs en terre d'Islam au Moyen Âge », Bulletin d'études orientales [En ligne], LXIV | 2016, mis en ligne le 01 avril 2018, consulté le 30 avril 2019 URL : http://journals.openedition.org/beo/4689; DOI : 10.4000/beo.4689 


\section{Entre fragrances et pestilences, étudier les odeurs en terre d'Islam au Moyen Âge}

Julie BONNÉRIC

"Le croyant qui récite le Coran est comme l'orange dont le goût est agréable et dont le parfum l'est aussi. Le croyant qui ne récite pas le Coran est comme la datte dont le goût est agréable et qui n'a pas de parfum. Le libertin qui récite le Coran est comme le myrte dont le parfum est agréable et la saveur amère. Le libertin qui ne récite pas le Coran est comme la coloquinte dont la saveur est amère et qui n'a pas de parfum. »

al-Buhārī, Kitāb al-ğāmi al-șahịh. ${ }^{1}$

Quoique l'Orient soit traditionnellement associé à un monde vibrant de senteurs parfumées et épicées, la question des odeurs en terre d'Islam à l'époque médiévale a peu intéressé les chercheurs ${ }^{2}$. Lorsqu'ils décrivent le raffinement de cette société médiévale, les historiens évoquent parfums, encens et onguents pour colorer leurs descriptions; ils en mentionnent la diversité et la qualité, ainsi que le coût faramineux et les origines lointaines. Si de très nombreuses sources, riches d'informations, sont pourtant disponibles, les analyses précises sont rares. À l'exception de deux articles de Françoise Aubaile-Sallenave qui s'intéressent spécifiquement aux odeurs ${ }^{3}$, ce thème a principalement été abordé de manière indirecte dans l'étude du commerce (AKASOY et YOELI-TLALIN 2007 ; CASEAU 2013 ; KING 2007) ou d'un produit odorant particulier - le musc (DIETRICH 1993), l'oliban (DieTRICH 1983), l'ambre (RUSKA et PlesSNER 1986 ; MotTAHEDIn 1985), le ban (AubaileSALLENAVE 1998), le camphre (A'LAM 1990, DieTRICH 1997 ; STÉPHAN 1998), le safran (WEINES et SANAGUSTIN 2002), le baume (MILWRIGHT 2003) ou l'eau de rose (GARCíA-SÁNCHEZ 1998).

1. Traduction O. Houdas et W. Marçais, Les traditions islamiques, Paris, 1914, vol. IV, p. 652.

2. L'auteur remercie vivement Frédéric Imbert, Lina Khanmé, Nadine Méouchy et Bruno Paoli sans qui ce numéro thématique n'aurait pas vu le jour, ainsi que les rapporteurs des articles pour leurs relectures et critiques.

3. Voir : Aubaile-SALLENAVE 1999 ; Aubaile-SALLENAVE 2004. 
Ces approches sont insuffisantes dans le cadre d'une histoire des odeurs, qui doit envisager le phénomène dans ses dimensions à la fois technique, sociale et symbolique. Une telle histoire nécessite une approche large et précise à la fois, réunissant historiens, linguistes, archéologues, spécialistes de droit ou de littérature.

Le présent dossier du Bulletin d'Études Orientales fait suite à une table ronde organisée par le Département des Études Arabes Médiévale et Moderne de l'Ifpo et à un appel à contribution plus large ${ }^{4}$. Il réunit des chercheurs qui, au-delà de la défiance que suscite habituellement la figure de l'odeur, ont exploité les sources dont ils sont spécialistes afin d'aborder un thème qui leur était, à quelques exceptions près, inconnu. Quatre axes principaux se détachent dans l'étude historique des odeurs, et ce sont ceux qui ressortent des différentes approches proposées ici: le vocabulaire des odeurs, les substances odorantes, les fonctions des odeurs et des odorants, le rapport des odeurs au sacré.

\section{Les odeurs, une histoire en formation}

Largement abordé, quoique tardivement, par les anthropologues, le thème des odeurs n'a pas reçu la même faveur de la part des historiens et des archéologues. À quelques exceptions près ${ }^{5}$, il n'a été exploré que de manière indirecte, à travers les parfums et leurs modes de fabrication, les matières premières requises pour la fabrication de ces parfums, la circulation des matières premières, les contenants à parfum comme les fioles et les encensoirs, ou les activités artisanales associées à la production d'odeurs déplaisantes. L'odeur elle-même est encore rarement étudiée comme fait historique, dans ses dimensions sociales et symboliques. Cette concentration de la recherche sur des aspects techniques, de production, de conservation ou de diffusion des parfums, qui ne traitent qu'indirectement des odeurs, s'explique aisément: d'une part, ce phénomène intangible, qui n'a pas de matière à proprement parler, est difficile à appréhender; d'autre part, les sources sont souvent hétéroclites et leur collecte est un obstacle à l'étude du phénomène. Certains chercheurs ont en outre perçu, dans cette absence d'intérêt de leur discipline pour les odeurs, une conséquence de leur dépréciation par un Occident moralisateur.

Pourtant, le thème des odeurs est d'un intérêt fondamental, en particulier parce qu'il revêt des dimensions à la fois sociales, culturelles et religieuses. Pour Pascal Lardellier, parfums et odeurs « pourraient à bon droit être considérés comme des “objets scientifiques

4. Cette table ronde, intitulée Fragrances et pestilences: histoire et anthropologie des odeurs en terre d'Islam à l'époque médiévale, a eu lieu le 14 décembre 2012 à l'Ifpo de Beyrouth. Y sont intervenus M. Balda-Tillier, J. Bonnéric, J. Candau, J.-Ch. Coulon, J.-Ch. Ducène, P. Koetschet, S. Le Maguer et B. Paoli. Que soient remerciés ici Bruno Paoli, alors directeur du DEAMM, ainsi que Isabelle Mermet et Amira Zakher, pour leur aide à l'organisation de cet événement.

5. L'étude du rapport du christianisme à l'odeur est sans doute la plus développée. Voir : ALBERT 1990 ; EVANS 2002 ; ASHBROOK HARVEY 2006. Sur l'Inde voir : MCHUGH 2012. Sur la période moderne (XVIII et XIX ${ }^{e}$ s) et son imaginaire social de l'odorat, voir : CORBIN 1982. Pour une approche transversale, voir le numéro 47 de la revue Terrain, qui a réuni historiens, anthropologues, linguistes et neurobiologistes pour travailler sur le rapport de l'homme à l'odeur (BALSAMO 2006). 
totaux" (...), car ils sont porteurs d'un sens pluriel et intégralement déclinés des points de vue étymologique et épistémologique, puisque tout à la fois sensoriels et sensuels, sémantiques et symboliques. ${ }^{6}{ }^{\prime}$

\section{Un phénomène intangible difficilement saisissable}

Toute la difficulté, nous l'avons dit, réside probablement dans la nature même de l'odeur, un phénomène labile, sans matière propre, et qui ne laisse par conséquent que des traces indirectes. Les textes ne décrivent ou ne mentionnent que rarement les odeurs, et les historiens ne disposent pour leur étude que d'allusions ou de mentions détournées, tandis que les archéologues n'en peuvent étudier que les à-côtés, c'est-à-dire les témoignages matériels indirects (ustensiles ou produits odorants). L'étude de ce phénomène volatile a néanmoins connu deux tournants: un premier, anthropologique et constitué par le Sensual Turn de Constance Classen et David Howes, assied l'odorat dans le champ d'étude de l'anthropologie (HOWES 1986; CLASSEN 1993 ; HOWES 2003 ; ClASSEN, HOWES et SYNNOTT 1994), ce thème n'étant apparu que ponctuellement dans la discipline comme l'explique Joël Candau dans ce volume ; le second, historique et probablement marqué par Le Miasme et la jonquille d'Alain Corbin (1982), aborde l'odeur comme phénomène historique global.

\section{Des sources hétéroclites}

Pour l'historien, comme pour l'archéologue, les sources relatives aux odeurs sont particulièrement hétéroclites. Les odeurs apparaissent - sous la forme d'expressions, d'allusions, parfois de descriptions brèves - dans des ouvrages aussi bien historiques et géographiques, que juridiques, scientifiques ou littéraires. Les données archéologiques sont également de nature variée et sont la plupart du temps relatives aux techniques de fabrication, de diffusion, de conservation ou de transport des produits odorants.

C'est sans doute cette difficulté à identifier et extraire les informations pertinentes dans l'ensemble particulièrement hétérogène des sources disponibles, qui explique, en histoire, le traitement uniforme de l'odeur, traitement réalisé sur des périodes très longues, conduisant à la formulation d'assertions intemporelles, souvent invariables. Considérons par exemple le thème des odeurs et du sacré : la bonne odeur - l'odeur plaisante - a été comprise comme un attribut essentiel du divin en Égypte, en Grèce et à Rome, sans que les différences, les spécificités et les évolutions dans cette association de la «bonne odeur » à la figure du divin n'aient été clairement systématisées ${ }^{7}$. Lorsque les odeurs sont évoquées, on se perd en effet souvent dans l'énumération d'anecdotes ou de récits mythiques.

\footnotetext{
6. LARDELLIER 2003, p. 11.

7. Voir en particulier LE GUÉRER 2005.
} 


\section{Le dégoût des odeurs}

Dès la Renaissance, une défiance généralisée à l'égard des odeurs, plaisantes ou déplaisantes, a touché le monde occidental et s'est poursuivie à l'époque contemporaine, tant chez les philosophes que chez les théologiens. Si le dégoût des odeurs peut s'inscrire, chez les religieux, dans la continuité d'une forme de défiance pour ce qui relève du corps et de ses productions, les senteurs sont très souvent associées par les philosophes à l'animalité et dégradées au rang de ce qui relève, contre le monde des Idées, de la dégradation et de la corruption des corps. Annick Le Guérer définit ainsi l'odorat: «Sens animal, primitif, instinctuel, voluptueux, érotique, égoïste, impertinent, asocial, contraire à la liberté, nous imposant, bon gré mal gré, les sensations les plus pénibles, inapte à l'abstraction, impuissant à sortir du solipsisme originaire de la subjectivité. ${ }^{8}$ » $\mathrm{Au} \mathrm{XX} \mathrm{X}^{\mathrm{e}}$ s., non seulement le corps mais également les odeurs sont réprouvés. Les espaces, publics ou privés, doivent en outre être non pas tant aseptisés que "reparfumés » afin de se soustraire aux odeurs naturelles. Évoluant eux-mêmes dans une "société de contrôle total des odeurs ${ }^{9}$ ", les historiens n'ont pas évalué l'intérêt de telles questions.

\section{En terre d'Islam}

Si l'étude des odeurs est ardue, concernant l'Islam médiéval comme d'autres civilisations, elle n'en est pas moins particulièrement riche car elle révèle des dimensions à la fois sociales et symboliques. Afin de répondre au mieux au caractère transversal des odeurs et au morcèlement des sources, ce dossier a dû réunir des chercheurs d'horizons divers, aux spécialités parfois très différentes, et dont le thème des odeurs en terre d'Islam à l'époque médiévale n'est pas nécessairement le sujet d'étude. Toute la difficulté de l'entreprise résidait donc, pour la majorité des auteurs, dans le traitement et l'usage «non familier » de sources «familières ». L'approche transversale promue dans ce volume fait apparaître quatre thèmes fondamentaux - le lexique des odeurs, les matières odorantes, les fonctions des odeurs et leur rapport au sacré - où les odeurs sont interrogées à la fois sous des aspects technique, social et symbolique. Avant d'aborder ces thèmes, précisons quelles sont les sources disponibles pour l'étude des odeurs et des odorants.

\section{Les sources et la nécessité d'une approche transversale}

Les articles réunis dans ce volume sont révélateurs de la diversité des sources pouvant renseigner une histoire des odeurs. Concernant les textes, presque tous les types de sources textuelles ont quelque chose à nous apprendre, tant sur les fragrances que sur les pestilences : textes littéraires, géographiques, historiques, juridiques, lexicographiques, médicaux, cosmétiques, botaniques, agronomiques, documentaires, magiques,

\footnotetext{
8. LE GUÉRER 2003, p. 17.

9. LARDELLIER 2003, p. 12
} 
philosophiques, religieux, etc. Bien que les informations soient souvent ténues, toutes les sources sont exploitables. Elles doivent même toutes être exploitées si l'objectif est de constituer une histoire des odeurs. Les traités de parfumerie, de médecine et de géographie, les plus couramment exploités, sont en effet insuffisants : certes les informations fournies sur les odorants sont très nombreuses, mais le rôle des odeurs n'y est pas réellement évoqué. En outre, les sources se complètent et se répondent: les hadiths, par exemple, apportent un point de vue théorique qui doit être confronté à celui des autres sources, en particulier littéraires. Ces dernières sont plus riches en informations sur le quotidien tel qu'il était vécu par la population et non pas tel que voulu par une élite religieuse ${ }^{10}$.

S'y ajoutent les sources archéologiques offrant une certaine matérialité aux odeurs. Les informations fournies sont techniques et concernent les odorants (analyse des résines) et les brûle-parfums, mais également sociales ou symboliques lorsque ces objets sont étudiés dans leur contexte d'utilisation. Afin d'améliorer notre compréhension du rôle des substances odorantes, il est important de porter plus d'attention à la fonction des objets. Les archéologues s'intéressent en effet principalement aux objets comme éléments de datation tandis que les historiens de l'art en apprécient les qualités stylistiques. Or, l'étude des brûle-parfums, mise en relation avec les textes, apporterait très probablement des éléments, non seulement sur la manière dont on procède aux fumigations, mais également sur le rôle social ou symbolique que peut revêtir l'encensement. Ainsi, dans son portrait des encens et de leurs usages, Sterenn Le Maguer a recours à ces deux types de sources. Néanmoins, elle est confrontée à l'absence d'objets en contexte, les archéologues mentionnant trop peu souvent la provenance exacte des objets. Une étude exhaustive de la littérature mentionnant les usages des brûle-parfums fournirait sans doute des éléments sur le sens accordé aux fumigations d'encens et, plus généralement, au rôle des parfums. Enfin l'iconographie, qui n'apparaît pas dans ce volume, pourrait sans doute s'avérer utile, notamment pour déterminer les contextes d'utilisation des odorants.

La seule approche permettant d'aborder les odeurs dans leurs dimensions historiques est transversale, non seulement parce que les données en sont enrichies et plus globales, mais également parce que les questions posées sont ainsi différentes. Une histoire des odeurs relevant pour partie de l'anthropologie historique, l'ensemble des articles de ce volume a été placé dans une perspective anthropologique grâce à l'article de Joël Candau, spécialiste d'anthropologie cognitive et sensorielle. Sa synthèse des données produites par l'anthropologie des odeurs est un état des lieux clarifiant certains points et relativisant des idées préconçues. Cette étude permet aux historiens et archéologues de confronter leurs données à l'analyse anthropologique et à ses grilles de lecture.

10. Voir, dans ce volume, les articles de A. Shinikov vs. M. Balda. 


\section{Exprimer les odeurs}

Un axe de recherche particulièrement riche est celui du rapport de la langue aux odeurs. Deux articles, l'un de Bruno Paoli sur l'arabe, l'autre de Jonas Sibony sur l'hébreu, y sont consacrés dans ce volume. Tous deux examinent le lexique des odeurs au prisme de la théorie des matrices et des étymons. Il en ressort que les étymons olfactifs sont relativement restreints dans les deux langues envisagées. Les perspectives sont néanmoins différentes : tandis que l'article de B. Paoli dresse un inventaire du vocabulaire olfactif à partir des dictionnaires, J. Sibony s'intéresse à un réseau sémantique particulier, à partir de la Bible hébraïque.

Bien que particulièrement riche, le lexique olfactif de l'arabe repose sur un nombre relativement limité d'étymons. B. Paoli ajoute six étymons aux dix étymons identifiés par George Bohas et Abdarrahim Saguer ainsi qu'une nouvelle matrice. Certaines racines discordantes pourraient, selon lui, correspondre à des variantes dialectales. Ainsi le verbe natuna, "sentir mauvais » pourrait provenir d'un *natuna, les dialectes arabes étant, semble-t-il, coutumiers de la transformation des interdentales en dentales. D'un point de vue linguistique, l'examen de ces étymons se révèle très enrichissant. Par ailleurs, la grille de lecture de l'auteur explique la variabilité d'évaluation d'une même racine. Ainsi, la racine [bhr], qui apparaît tout au long de ce volume (dans bahūur, " encens », ou mabhara, "brûle-parfum »), est liée aux mauvaises odeurs (bahrr, "mauvaise haleine », bahira, " sentir mauvais ») et aux bonnes (bahūr, mabhara, etc.). Le linguiste situe cette racine à la croisée de deux étymons, $\{b, h\}$ et $\{b, r\}$, le premier étant lié à des verbes de type «sentir mauvais », le second à des verbes de type « parfumer ».

L'article de B. Paoli s'insère dans un débat large, à la fois linguistique et anthropologique, sur la pauvreté intrinsèque de la langue dans l'expression des odeurs et de l'odorat, idée nuancée par J. Candau dans ce volume. L'arabe est en effet particulièrement riche avec environ 400 mots au sens olfactif. Cette richesse serait-elle le reflet d'une culture de l'odeur, à mettre en relation avec le rôle des marchands arabes dans le transport des substances odorantes? Nous évitant de formuler des interprétations hâtives sur cette profusion lexicale, l'auteur tempère cette richesse lexicale, en révélant la polysémie de la plupart des racines et leur synonymie. En effet, seules 21 racines, sur les 160 qui servent de base à ce lexique, n'ont qu'un sens olfactif, les autres étant polysémiques. Il faut par ailleurs garder à l'esprit la synonymie importante de ce lexique, ses différents termes n'apportant pas de nuances de sens très importantes. Le champ des sens exprimés est en effet relativement restreint. L'auteur suppose que les dictionnaires arabes ont probablement réuni arabe classique et vocabulaire dialectal et que la richesse du lexique pourrait résulter de la multitude de dialectes présents en Arabie. Quoiqu'il en soit, ce qui distingue principalement ce riche lexique est une variable d'évaluation entre mauvaises (77 racines) et bonnes odeurs (moins de 30 racines), les secondes étant beaucoup moins développées que les premières. Cette prééminence rejoint les observations générales de la linguiste Claude Boisson (1997), qui a tenté d'établir des régularités et des variétés linguistiques dans la dénomination des odeurs. Enfin, à la suite de G. Bohas et A. Saguer (2012), B. Paoli motive l'importance des 
consonnes nasales et continues dans les étymons olfactifs par un symbolisme de la nasalité et de la continuité.

Souhaitant analyser le champ sémantique de l'odeur, J. Sibony met également en évidence les liens tissés entre sons et sens en hébreux, en l'occurrence entre des éléments phoniques semblables et les notions d'odeurs, de souffle et de sacré. Bien qu'il s'appuie également sur la théorie des étymons et des matrices, l'auteur développe une autre perspective que celle de B. Paoli, celle de la sémantique lexicale. Il procède à une enquête étymologique afin d'expliquer cette corrélation phono-sémantique entre similitudes acoustiques ou articulatoires et champ conceptuel. L'analyse des racines et des étymons en lien avec, tour à tour, le souffle, les odeurs, la vie, l'âme et le sacré, révèle que souffle, odeur et sacré ne sont en réalité que différentes facettes exprimant la même idée. Or, formuler une idée polysémique par des mots construits sur un même modèle phonique fait sens. Certains termes relatifs aux odeurs et qui ne peuvent être associés phonétiquement aux étymons définis par J. Sibony, sont rapprochés par ce dernier, à partir de leur contexte d'usage, d'un autre champ lexical, celui du sacrifice et de la coupure.

L'approche linguistique du thème des odeurs est, on le voit, particulièrement féconde pour la compréhension des représentations mentales des sociétés étudiées. En outre, les articles de B. Paoli et J. Sibony n'apportent pas seulement à leur discipline, celle de la linguistique sémitique, mais contribueront également à faire avancer l'un des trois axes définis par J. Candau comme étant les plus dynamiques dans l'anthropologie des odeurs, celui de la description et de la catégorisation des odeurs puisqu'ils enrichissent le corpus de descripteurs culturels.

\section{Substances odorantes}

Contrairement à l'odorat et à l'odeur, les matières odorantes ont retenu l'attention des chercheurs, probablement du fait de leur matérialité et des nombreuses sources qui les documentent. Néanmoins, les études approfondies sont encore rares ${ }^{11}$. Ce volume révèle la variété des produits odorants, simples et complexes. Les matières simples, nombreuses, sont en effet très souvent mélangées en compositions plus ou moins subtiles. Certains parfums comme la gäliya, le nadd ou le haluq sont particulièrement célèbres et composés de nombreuses substances de valeur. Les odorants sont multiples du fait de l'étendue de la gamme employée et de la diversité des provenances. Ces produits sont en effet des matières animales, des résines, des écorces, des feuilles, des fleurs ou encore des racines. Simples ou composés, ils sont utilisés bruts, à brûler, ou après macération, cuisson, décantation, distillation, sous forme d'huiles, d'eaux parfumées, d'onguents, d'encens, etc. Issus d'une production locale, ils sont originaires de toutes les régions de l'empire ou, fruits d'un

11. À l'exception de la thèse d'Anya King (2008) sur le musc, de l'article de Françoise Aubaile-Sallenave (1998) sur le bân, de l'article de Noucha Stéphan (1998) sur le camphre et de l'article d’Expiración García-Sánchez (1998) sur l'eau de rose, les autres publications sur des substances odoriférantes sont des articles d'encyclopédie, souvent denses mais peu approfondis (MOTTAHEDIN 1985 ; RUSKA et PLESSNER 1986 ; DIETRICH 1983 ; ÁLAM 1990 ; DIETRICH 1993 ; DiETRICH 1997 ; WEINES et SANAGUSTIN 2002, MILWRIGHT 2003). 
commerce à longue distance, de régions exotiques, à la fois orientales et occidentales. Dans son Kitāb ğawāhir al-țīb al-mufrada, Ibn Māsawayh dénombre quatre-vingt-six substances odorantes (LEVEY 1961). La variété des produits à brûler recensés par Jean-Charles Ducène est également représentative. Si certains sont d'origine animale - musc (misk), ambre ('anbar) et blattes de Byzance (azfār al-tịib) -, la majorité est végétale: oliban (lubān ou kundur), camphre (kâfür), myrrhe (murr), bois d'agalloche ('ūd), santal (șandal), styrax (may'a), baume de Judée (balasān), acacia (umm ġaylān), sandaraque (sandarūs), ladanum (lādan), mastic (maștaka). En outre, beaucoup de plantes séchées exhalent, au moment de leur combustion, des senteurs appréciées : narcisse (narğīs), rose (ward) ou rose musquée (nasrīn), myrte (ās), jasmin (yasmīn), menthe (namām), citron (laymūn), cédrat ('utruğ), etc. Certaines écorces brûlées, comme celles du grenadier (rummān), du cognassier (safarḡāl) et du teck (sāğ), sont recommandées par les médecins parce qu'elles purifient l'air. L'inventaire des produits odorants utilisés en magie, dressé par Jean-Charles Coulon, est également édifiant. Bien que l'empire arabo-musulman couvre à la période médiévale des zones productrices de multiples substances odorantes, un intérêt particulier est porté aux senteurs exotiques, comme le remarquent à la fois S. Le Maguer et J.-Ch. Ducène ${ }^{12}$. Lorsque les textes désignent précisément la substance et ne recourent pas au terme générique de bahūur, ce sont le musc (misk), l'agalloche ('ūd) et l'ambre ('anbar) qui sont le plus fréquemment mentionnés, tandis que l'encens oliban (lubān ou kundur) apparaît relativement peu. Cela se confirme dans les textes magiques présentés par J.-Ch. Coulon. Comme le note S. Le Maguer, l'oliban n'apparaît même pas dans la liste des principales substances aromatiques (al-'ușūl) établie par Ibn Māsawayh au IX ${ }^{\mathrm{e}} \mathrm{s}$. Les matières les plus employées sont d'origine lointaine : l'agalloche est probablement originaire de l'Inde et d'Asie du Sud-Est, le musc d'Asie centrale, et l'ambre du Yémen, d'Oman, d'Afrique orientale et, dans une moindre mesure, de la partie occidentale de l'empire. L'oliban en revanche, bien que produit également en Inde, est particulièrement répandu dans le Sud de l'Arabie et la corne de l'Afrique.

L'un des odorants les plus répandus à l'époque médiévale, l'ambre gris ('anbar), est l'objet de l'article de Thierry Buquet qui s'intéresse plus particulièrement à l'origine - animale, végétale ou minérale - que lui attribuent les auteurs arabes médiévaux. De nombreuses sources sont compulsées afin de dresser un portrait complet des hypothèses formulées. Pour certains auteurs, l'ambre serait une plante marine (sorte de champignon ou de truffe), voire un liquide minéral sous-marin, qui serait rejeté hors des eaux lors de tempêtes, autorisant ainsi sa récolte. Bien que l'ambre gris soit en réalité une concrétion intestinale du cachalot, les théories liées à la baleine paraissent minoritaires. Pour leurs tenants, l'ambre est un produit dont les propriétés se déploient après digestion de la baleine qui l'aurait avalé. Il peut alors être ramassé sur la plage ou dans le ventre même de l'animal dont il causerait la mort. Une variante de cette explication est celle d'une déjection de la baleine.

12. Un article récent se concentre sur ce thème : LEV et ZOHAR 2013. 
L'origine naturelle de l'ambre, ainsi que sa provenance géographique ou sa couleur, sont des critères d'évaluation de sa qualité. L'ambre de baleine aurait une odeur de poisson et se trouve donc dévalorisé. L'ambre le plus recherché provient de l'océan Indien (côtes du Yémen, d'Oman, côte orientale de l'Afrique), la qualité de l'ambre occidental (Maghreb, Espagne, océan Atlantique) ne faisant pas l'unanimité. Les auteurs paraissent relativement unanimes sur la valeur de l'ambre de couleur blanche. Les ambres tachetés ou jaune et rouge seraient en revanche de mauvaise qualité. Les auteurs ne fournissent pas de détail sur les différentes qualités odorantes de l'ambre et seul apparait le rejet de l'ambre dite samakiyya, à l'odeur de poisson. Lorsque l'ambre n'a pas la couleur adéquate, qu'il sent le poisson ou qu'il n'est pas frais, il est inadapté à la parfumerie.

L'odeur de l'ambre, qui est pourtant la raison d'être de son usage, n'est donc pas le critère principal de classification de ce produit. Néanmoins, c'est bien la fragrance de l'ambre qui en constitue la valeur, l'objectif étant d'améliorer les parfums. Les auteurs, bien qu'ils aient conscience, pour certains, de l'origine animale de l'ambre et bien que la fraîcheur de l'ambre soit parfois évoquée, n'ont pas compris que les fragrances de l'ambre s'exhalaient après exposition de la substance à la lumière, pendant plusieurs mois, s'améliorant au rythme de l'exposition. Qu'ils soient médecins, encyclopédistes ou géographes, ils préfèrent reconstituer une origine fabuleuse de l'ambre, offrant ainsi l'occasion d'un récit parfois merveilleux, plutôt que d'observer un phénomène relativement trivial. La couleur n'en demeure pas moins un bon diagnostic puisqu'elle témoigne de la durée d'exposition de l'ambre et donc de sa qualité olfactive : noirâtre à l'origine, il s'éclaircit ensuite du gris argent au jaune or et jusqu'au blanc jaunâtre. N'est-il pas étonnant que l'odeur même de l'ambre ne soit pas le critère distinctif de sa qualité ? Les odeurs sont néanmoins difficiles à mettre en mots, et la langue arabe, comme en témoignent le lexique et les textes littéraires, ne favorise pas la description des notes des produits odorants ${ }^{13}$.

Tandis que l'article de Th. Buquet est uniquement construit à partir de sources textuelles, celui de S. Le Maguer illustre tout à fait la nécessité de l'interdisciplinarité. Afin de dresser un portrait de l'encens et de ses usages du VIII ${ }^{\mathrm{e}}$ au XII ${ }^{\mathrm{e}} \mathrm{s}$, l'auteur fait appel à des sources multiples - archéologiques, physico-chimiques et textuelles, qu'il s'agisse de traités de médecine, d'ouvrages de géographie ou de littérature. Les données archéologiques sont principalement fournies par les brûle-parfums. Les substances odorantes elles-mêmes sont rarement mises au jour, soit parce qu'elles se conservent mal, soit parce que les archéologues recourent rarement au tamisage. Pourtant sur le site de Qasr Ibrīm en Nubie, seul le tamisage des sédiments a autorisé la découverte de résines. Ces matériaux documentent la nature de l'encens ou sa composition. Sur ce même site, les analyses ont ainsi révélé que l'encens employé était un mélange d'oliban (de l'encens au sens propre) et de résine de pin (EVERSHED et al. 1997). Comme le précise S. Le Maguer, si les analyses ne permettent pas de retracer l'origine des produits, elles offrent néanmoins des indices sur les trajets parcourus par ces produits et sur leur commerce. Fabriqués en céramique, en

13. La note est une caractéristique olfactive par laquelle est décrit un parfum. 
pierre et en métal, les encensoirs ont quant à eux laissé plus de traces archéologiques. Les brûle-parfums en céramique sont les plus variés car leur production est locale et diversifiée. Les encensoirs en pierre sont taillés dans de la chlorite ou de la stéatite. L'Arabie est une zone d'extraction importante de ce type de pierres, tout comme de production de brûleparfums. Les découvertes archéologiques attestent leur importation en Irak et en Iran, mais également dans le Bilād al-Šām et en Égypte. La forme paraît relativement standardisée avec un récipient circulaire ou quadrangulaire supporté par des pieds, souvent orné d'un décor géométrique incisé et présentant un manche horizontal. Les encensoirs en métal sont réalisés dans un alliage de bronze ou de cuivre et prennent fréquemment la forme d'un récipient cylindrique ou circulaire, reposant sur des pieds (souvent trois) et couvert par un couvercle ajouré, l'objet étant manipulé à l'aide d'un long manche horizontal. La production seldjoukide iranienne est bien documentée et se compose de brûle-parfums zoomorphes (lions, oiseaux), souvent monumentaux. Quatre termes les désignent: mabhara, mağmara, madhan ou maqțar. S. Le Maguer n'est malheureusement pas parvenue à les mettre en relation avec des objets spécifiques, en fonction de leurs matériaux de fabrication, de leur forme ou du produit qui y était brûlé. Leur racine seule pourrait laisser penser que la mağmara est destinée à parfumer les vêtements et le maqțar à brûler spécifiquement de l'aloès. Rien ne confirme néanmoins la réalité des pratiques indiquées par leur étymologie. Une étude contextuelle, précisant le cadre dans lequel chaque terme est employé, offrirait peut-être des informations complémentaires.

L'étude des brûle-parfums permet de dresser une typo-chronologie de ces objets, qui deviennent alors diagnostiques pour l'archéologue (LE MAGUER 2011), ainsi que de compléter la carte des échanges de produits odorants. Associée au contexte de découverte des objets, cette étude se révèle en outre essentielle à l'histoire des odeurs et pas uniquement des odorants. Les brûle-parfums pallient le silence des sources puisqu'ils documentent des milieux rarement évoqués par les textes. Ainsi, la présence récurrente de brûle-parfums en céramique dans des contextes domestiques modestes atteste une certaine banalité de l'encensement de foyers peu fortunés, probablement grâce à un mélange de substances odorantes de moindres qualité et coût.

\section{Le rôle des odeurs}

La question des odeurs est une question globale puisque celles-ci prennent part à l'ensemble des activités humaines. Les articles de ce volume documentent la thématique centrale des usages et fonctions des odeurs. J.-Ch. Ducène dresse en particulier un portrait d'ensemble des différents usages des fumigations, principalement à partir de sources géographiques et historiques. Comme les huiles parfumées ${ }^{14}$ et autres produits odoriférants, l'encens est employé en parfumerie et en médecine. En revanche, c'est lui qui est privilégié dans le cadre cérémoniel (usages domestiques, princiers ou sacrés),

14. Pour une étude des différents usages des huiles, voir BONNÉRIC 2012. 
pour parfumer à la fois les vêtements et l'ambiance. J.-Ch. Coulon se concentre sur le rôle des odeurs, en particulier de la fumigation, dans la magie arabe médiévale. Monica Balda s'intéresse quant à elle, à partir de textes littéraires, à l'usage des parfums et aux mauvaises odeurs, et en particulier à la valeur morale qu'ils peuvent revêtir. Les ouvrages de son corpus sont des traités d'amour ou de poésie amoureuse au travers desquels sont enseignées les règles de comportement en société d'une élite culturelle et sociale. Si ce volume n'examine pas tous les usages, il offre néanmoins une vue relativement large des fonctions culturelles, sociales et symboliques des odeurs. Passons en revue ces principaux usages, à savoir cosmétiques, médicaux, culinaires et cérémoniels, qui nous informent sur le rôle des odeurs.

Bien entendu, l'usage des produits odorants en cosmétique et en parfumerie est évident, mais il est particulièrement développé en Islam à la période médiévale, avec l'utilisation de nombreux produits. Un soin particulier est apporté, grâce à des produits parfumés variés, au corps, à la fin du bain en particulier, aux cheveux et au visage (BESSARD 2008, BONNÉRIC 2012). Concernant le soin des cheveux, sont notamment mentionnées l'huile (duhn) de myrte ('ās), de giroflée (hìrì), d'azédarac (azādraht), de violette (banafsağ), de cédrat ('utruğ) ou encore de jasmin (yāsmīn). Certaines huiles fortifient ou embellissent les cheveux, d'autres stimulent leur croissance, d'autres préviennent leur chute. Concernant les soins esthétiques, les huiles parfumées ne sont pas employées de manière indifférenciée, comme c'est probablement le cas en médecine, et les indications fournies reflètent peut-être ici des usages réels. Néanmoins, ce n'est pas l'odeur qui importe, mais les propriétés des plantes, qu'elles soient odorantes ou non. Pour traiter des odeurs, l'usage cosmétique le plus intéressant est donc, et cela n'a rien d'étonnant, la parfumerie. Pour se parfumer, les deux principales pratiques étaient les fumigations pour embaumer vêtements et cheveux et l'application de parfums (huiles ou onguents aromatisés) sur la peau. Des pastilles parfumaient en outre l'haleine. Les femmes recouraient à de nombreux parfums (țīb ou 'ițr), qu'ils soient composés (halūq, nadd, barmakiyya, etc.) ou simples (par exemple santal, safran, camphre, violette). Les hommes également emploient des parfums, mais leurs choix paraissent plus limités (M. Balda, A. Shinikov). D’après le Kitāb al-Muwaššă d'al-Waššă' (m. 325/937), tous les parfums ne sont pas licites pour les hommes raffinés (zurafä'). Ainsi, tandis que le musc (misk), l'ambre ('anbar), l'agalloche ('ūd) et le safran ${ }^{15}$ ('abir) sont autorisés, d'autres sont illicites ou ne peuvent être employés que dans des cas particuliers. Le camphre ( $k a \overline{f u}$ r $)$ est déconseillé pour des raisons médicales et, suivant la théorie des humeurs, n'est autorisé qu'en association avec du safran et du musc, ces derniers régulant son humeur froide, ou alors lorsqu'il fait très chaud. Certains parfums composés sont interdits afin de distinguer les personnes raffinées d'autres catégories de la population. La barmakiyya ne peut être employée car elle l'est déjà par des "canailles" (mutaqallitūn). Le halūq, ce parfum à base de safran, est quant à lui associé aux femmes.

15. L'interprétation de M. Balda a ici été conservée bien que ce terme pourrait désigner plus génériquement un parfum (voir note 26). 
La gālia, bien que composée de musc et d'ambre, est réservée aux jeunes garçons (șibyān) et aux esclaves femmes (ima $\bar{a}$ ). Enfin, la couleur du parfum a ici encore son importance puisque le dafir ne peut être employé du fait qu'il marque la peau et les vêtements. Al-Wašša en effet fait référence à une tradition prophétique réclamant que le parfum ne tâche pas. Comme le fait remarquer M. Balda, les explications d'al-Waššă' signifient que l'odeur distingue les individus selon leur genre ${ }^{16}$ et leur appartenance sociale. Bien entendu, ce sont les parfums les plus onéreux qui constituent un marqueur social. Notons que si les esclaves chanteuses et les éphèbes sont dépeints par leurs odeurs enivrantes, ce n'est que pour mieux valoriser leur maître. Certes les parfums mentionnés dans les textes sont réservés à une élite fortunée, mais certaines huiles simples comme l'huile de violette (duhn al-banafsağ), mentionnée par al-Ǧāhị (m. 255/868), ou l'huile de cédrat (duhn al-'utruğ) recommandée par Ibn al-Bayțār, étaient peut-être accessibles à des milieux plus modestes, marchands, commerçants, artisans spécialisés (BONNÉRIC 2012).

En médecine, l'emploi de produits odorants est très développé, en potions, onctions, injections, inhalations ou fumigations ${ }^{17}$. L'objectif est bien sûr de lutter contre des maladies, mais également de purifier l'air (J.-Ch. Ducène). Les prescriptions dépendent de la théorie aristotélicienne des humeurs, le choix des substances se faisant en fonction de leur nature, froide, chaude, humide ou sèche, et de celle de la maladie à traiter ou de l'air ambiant. Pour purifier l'air, les fumigations sont privilégiées. Les médecins recommandent des produits très variés, moins luxueux qu'on pourrait le croire, donc peut-être plus répandus : bois de genèvrier ('ar'ar), de laurier ( $\dot{g} \bar{a} r)$, de cyprès (sarw), etc. En revanche, les huiles ou autres produits odorants ont une vocation thérapeutique plus marquée, tandis que les fumigations sont peu employées, si ce n'est pour les problèmes respiratoires. Là encore, les produits employés sont très variés. Prenons l'exemple des problèmes sexuels, contre lesquels les huiles sont un recours particulièrement prisé ${ }^{18}$. Contre les problèmes d'érection, al-Dimašqī recommande ainsi l'inhalation d'huiles froides et humides comme l'huile de violette (duhn al-banafsağ) pour les tempéraments chauds, et l'inhalation d'huiles chaudes et humides comme l'huile de jasmin (duhn al-yāsmīn) pour les tempéraments froids. L'huile de giroflée (duhn al-hīìi) et l'huile de moutarde (duhn al-hardal) renforcent la puissance sexuelle et stimule le coït. Les huiles peuvent être appliquées sur la verge (duhn al-hīîi) ou en lavement (huile de chardon étoilé, duhn al-hasak, ou huile de coco, duhn al-nārğil). En revanche, l'huile de violette (duhn al-banafsağ) injectée dans la verge permet de calmer les ardeurs de la verge et de la vessie. La richesse des huiles parfumées disponibles et le fait que les huiles ne soient pas employées pour un trouble spécifique,

16. Ce thème a été rapidement abordé par Hadas Hirsch (2013).

17. Un Épitre sur le parfum écrit par Avicenne ( $\mathrm{x}^{\mathrm{e}} \mathrm{s}$.) justifie même un hadith relatant l'amour du Prophète pour les parfums par des raisons de santé : les parfums auraient une action positive sur l'esprit qui s'épuise à partir d'un certain âge. Voir MicHOT 1978 (traduction, édition et petit commentaire). Sur les parfums et la pharmacologie, voir SANAGUSTIN 1998.

18. Ces exemples sont tirés de la Risāla al-Hārūniyya de Masīh b. Hakam al-Dimašqī. Elle a été éditée et traduite par Suzanne Gigandet (2002). 
laissent penser que la prescription ne dépendait pas réellement de vertus thérapeutiques mais plutôt d'une sorte de démonstration d'érudition de la part du médecin qui laissait ainsi supposer l'étendue de ses compétences ${ }^{19}$. Enfin, tandis que, pour les fumigations, c'est bien l'odeur qui est sensée avoir un effet thérapeutique, pour les onguents, huiles, pilules et potions parfumés, il semble que ce ne soit pas l'odeur émise qui produise un effet thérapeutique mais plutôt les propriétés des plantes odorantes.

Les odeurs jouent bien entendu un rôle primordial en cuisine, puisqu'elles ajoutent à la saveur du plat. N'y étant pas abordé, ce thème constitue l'une des lacunes du volume. Pourtant les épices, substances odorantes s'il en est, sont des éléments essentiels de la cuisine ${ }^{20}$. Elles entrent dans la composition de presque tous les plats. Les traités de cuisine, comme le Kitäb al-țabīh ( $\mathrm{x}^{\mathrm{e}} \mathrm{s}$.), lui consacrent des chapitres spécifiques. Les épices, comme les médicaments, sont classifiés suivant la théorie des humeurs (le poivre noir, la coriandre et le cumin sont par exemple des épices chaudes) et les traités de cuisine, comme de médecine, réglementent leurs usages notamment à partir de cette classification. Un calendrier alimentaire suivant cette théorie est recommandé par les médecins : al-Dimašqī recommande les aromates et parfums chauds et secs en hiver mais chauds et humides en automne. Les épices ne sont pas les seuls éléments mentionnés par les traités de cuisine comme parfumant les plats. L'huile d'olive, par exemple, est également louée pour cette raison (BONNÉRIC 2012), le gras paraissant faire ressortir les saveurs. Les huiles et les eaux parfumées sont également employées pour améliorer les qualités gustatives, en particulier des desserts (eau de rose et huile d'amande principalement). Ainsi, le maḥlab al-hășșa al-sulțānī, d'après l'une des recettes du Kitāb al-țabĭh, nécessite de l'huile de jasmin de deux sortes différentes (zanbaq färisī et duhn al-zanbaq al-sābūrî̀), de l'huile de rose perse (duhn ward färisī), de l'huile de baume (duhn balasān), de l'huile de cédrat (duhn 'utruğ) et de l'eau camphrée $\left(m \bar{a}{ }^{\prime} k \bar{a} f \bar{r} r\right)^{21}$. Bien entendu, cette cuisine est celle d'une élite, la majorité de la population devant se contenter d'une nourriture plus frustre ${ }^{22}$.

Les fumigations, de bonnes ou mauvaises odeurs, constituent un pilier des pratiques magiques, comme le démontre J.-Ch. Coulon, car elles répondent au système d'analogie suivant lequel ces rituels fonctionnent. Elles sont associées aux djinns et aux esprits avec lesquels elles facilitent la communication, ainsi qu'aux astres car ceux-ci sont liés aux esprits célestes. Les fumigations reposent sur un système de correspondance astrale. Le recours à des références religieuses (Bouddha ou Moïse) légitime ces usages. Les bonnes odeurs agissent positivement dans le cadre de purifications et de guérisons tandis que les mauvaises odeurs répondent à des visées sinistres et nuisibles comme rendre l'esprit ou le corps malades. Les astres sont mis en relation avec de nombreuses substances aromatiques,

19. BONNÉRIC 2012, p. 308.

20. Sur les substances odorantes, en particulier les épices, voir les articles de Manuela Marín (1998), Expiración García-Sánchez (1997) et Françoise Aubaile-Sallenave (1994). Sur les épices et leur commerce, voir : FISCHEL 1953.

21. D’après la traduction en anglais : NASRALLAH 2007, p. 497.

22. Probablement du pain de froment (puis de riz), des fruits et des légumes et, à l'occasion, du poisson ou de la viande de bœuf, de cheval, de chameau suivant la région (ASHTOR 1968). 
comme l'ambre gris ('anbar), le musc (misk), le camphre (kāfür), le safran (za'farān), le santal jaune (șandal aṣfar) et le fenouil (basbāsa) pour Jupiter. D'un auteur à l'autre, les correspondances ne sont pas tout à fait semblables. D'autres substances composent des fumigations aux astres dont J.-Ch. Coulon fournit des exemples dans ce volume. En correspondance avec les planètes, les odeurs, en particulier dégagées par des fumigations, prennent en outre place dans l'élaboration de talismans.

En botanique et en agronomie, les odeurs, bonnes ou mauvaises, sont des éléments d'évaluation importants de l'état des cultures. L'article de Saadi Chakhoum et Hayat Guenoune se concentre sur les mauvaises odeurs. Elles indiquent en effet la qualité de l'engrais qui doit être appliqué sur les plantes. Les engrais sont constitués de fumier et d'excréments putréfiés. Suivant la nature du matériau (excréments humains, de chèvre, de mouton, d'oiseaux, etc.) et l'état d'avancement de la putréfaction, la qualité de l'engrais varie. Les agronomes et botanistes andalous sur lesquels s'appuient les auteurs emploient une terminologie précise et décrivent le processus de la décomposition afin de mieux coter les qualités d'engrais. Plus la décomposition est longue, meilleure sera la qualité de l'engrais. D'après les auteurs anciens sur lesquels s'appuient les agronomes andalous, il est recommandé de laisser vieillir trois ou quatre ans l'engrais, qui sera alors plus lisse et moins odorant, la pestilence constituant une sorte de mesure expérimentale. La noirceur de l'engrais est aussi un indice.

Les fragrances participent au cérémonial, qu'il soit domestique, princier ou sacré. Les textes témoignent de ces pratiques, parfois en les condamnant, mais ne fournissent néanmoins que peu de détails sur les matières odorantes et sur le processus de diffusion des senteurs. L'encensement est la méthode la plus courante pour embaumer les cérémonies, car les effluves se diffusent dans l'espace, agrémentant l'ambiance et parce qu'il dégage des senteurs plus profondes. L'aspect théâtral des fumigations lorsqu'elles sont pratiquées par des thuriféraires qui agitent un brûle-parfum portatif et produisent de la fumée, joue sans doute un rôle dans l'attrait pour l'encensement. Le recours à des luminaires parfumés est également attesté (J.-Ch. Ducène, J. Bonnéric). Concernant les usages domestiques, les textes littéraires en particulier témoignent de l'usage de fumigations dans les demeures de haut rang ou de marchands. L'archéologie révèle par ailleurs que même des habitats plus modestes pouvaient être embaumés (S. Le Maguer). Dans les maisons, l'objectif était non seulement de purifier l'air suivant des recommandations thérapeutiques, mais également d'accueillir et parfumer les visiteurs. Le calife s'entourait lui aussi de fragrances. Les historiens et géographes décrivent de multiples cas d'encensement d'audiences, de processions, de cérémonies (J.-Ch. Ducène). Quant à la fumigation en contexte sacré, c'est une question plus complexe. Comme le constate J.-Ch. Ducène, il n'y a pas d'islamisation de l'encens et plus généralement des produits parfumés. Les traces d'usage de substances odorantes dans les mosquées sont très restreintes et, si elles indiquent que ces produits n'en étaient pas bannis, les sources laissent supposer qu'elles n'étaient pas non plus utilisées pour le rite (J. Bonnéric). D’autres cérémonies à caractère religieux recourent aux encens, en particulier les cérémonies mortuaires (DE GOEJE 1907 ; A'LAM 1990) et soufies 
(J.-Ch. Ducène). Les morts peuvent en effet être honorés par des fumigations, d'ailleurs condamnées par l'orthodoxie d'Ibn Taymiyya (m. 728/1328). Ils peuvent en outre être lavés à l'eau parfumée ${ }^{23}$. Les soufis recourent quant à eux à l'encens lors des séances de $\underline{d} i k r$, durant les prières et les litanies. Contrairement aux mosquées, un waqf mentionnant le bahūr est attesté. Les exemples d'usages modernes dans le cadre du culte des saints sont nombreux (dans ce volume, voir l'article de S. Le Maguer). Les fumigations ou onctions aromatisées s'expriment dans le cadre de la piété populaire. L'orthodoxie en revanche les dédaigne, comme l'illustre le cas des grandes mosquées, voire les condamne. Les sources sont trop lacunaires pour examiner le cas des petites mosquées de quartier ou de village.

\section{Symbolisme}

Si les odeurs constituent des marqueurs sociaux et peuvent être un moyen de signifier un statut social, c'est parce que les fragrances sont des signes de qualités morales et les pestilences de débauche et de perversion, des individus comme des villes. Les textes littéraires en particulier confirment l'association entre mauvaises odeurs et bassesse, laideur, misère, infamie, en opposition aux bonnes odeurs, symboles de pureté et de propreté. Ainsi, le héros de la Hikāyat Abi al-Qāsim al-Bag̉dādī va même jusqu'à affirmer que Bagdad fleure l'ambre et que son sol exhale des senteurs de musc parfumé à l'eau de rose au matin. Tandis que les fragrances bagdadiennes symbolisent la pureté de la ville, de ses habitants et de ses institutions, la pestilence d'Ispahan, où les effluves de fumier rivalisent avec les excréments qui s'amassent dans les rues, reflètent la bassesse et la corruption de son élite (M. Balda). Par ailleurs, les insultes liées aux mauvaises odeurs sont variées: odeurs des excréments animaux ou humains, du sexe associé à l'insalubrité, des aliments réputés malodorants, des puanteurs du pourrissement, des pestilences professionnelles liées à des métiers malodorants, etc. Ces insultes, caricaturales et relevant du registre comique, évoquent les vices et les mauvaises mœurs (M. Balda). Elles signifient l'indignité des élites et leur abus de pouvoir. Enfin en magie, les actions positives (guérisons) exigent des senteurs parfumées tandis que les actions négatives (rendre malade, assassiner) requièrent des exhalaisons fétides (J.-Ch. Coulon).

En Islam, les odeurs ont une valeur métaphorique particulièrement forte. Dan Sperber (1974) explique ce phénomène général, et non spécifique à cette culture, par le recours inévitable à la métaphore pour décrire les odeurs, par leur pouvoir d'évocation et par l'impossibilité à se les remémorer ${ }^{24}$. En arabe et en hébreu, odeur et esprit sont étymologiquement liés (J. Sibony) : en arabe, rä̉iha, l'«odeur», est formé sur la même racine que rūh, l'« esprit», en hébreux, $r \bar{e}^{a} h$ et $r \hat{u}^{a} h$ (" souffle, vie, esprit»). Dans cette dernière langue, les étymons olfactifs sont associés phonétiquement, soit au champ lexical du souffle, de l'âme et du sacré, soit à celui du sacrifice et de la coupure. En effet, « symboles

23. TRITTON 1937, p. 653.

24. Sur le symbolisme des odeurs, voir l'approche anthropologique d'Alfred Gell (2006) ou l'ouvrage historique de Marcel Détienne (2007). 
par excellence ${ }^{25} »$, les odeurs entretiennent un lien étroit avec le sacré. Certaines odeurs, comme celles du musc ou de l'ambre qui sont caractéristiques du Paradis, sont valorisées tandis que d'autres sont prohibées. Les odeurs entrent dans la définition du halāl et du ḩarām. Ainsi, si le safran peut être théoriquement interdit aux hommes, c'est parce que son odeur et sa couleur portent une signification transcendantale.

En effet, une prohibition pour les hommes de s'appliquer du safran est transmise par les hadiths. Néanmoins, comme le révèle Atanas Shinikov dans ce volume, le contexte dans lequel cette interdiction est émise rend son interprétation difficile. Elle est en effet précédée d'une référence aux vêtements (tawb) et suivie d'une proscription pour les personnes en état d'ihrām (sans précision de genre) de porter des vêtements teints au safran. Pourtant, certains juristes considèrent qu'un habit teint au safran est autorisé et que seul le muhrim doit se préserver de ce produit. D'autres en revanche estiment que cette défense doit être respectée, que l'on soit en état d'ihrām ou non. D'après le juriste šâfi'îte Ibn Hağar, il semble que la majorité des exégèses sunnites penchent pour la proscription du safran aux hommes, les femmes ne subissant pas cette prohibition. Le même auteur justifie cette interdiction justement parce que le safran entrerait dans la composition de parfums pour femme et parce que le halūq, un parfum à base de safran, est défendu également. Une autre explication pourrait être, d'après lui, la couleur jaune de cette matière. En effet, le safran est à la fois un produit odorant (țîb) et colorant (șibg) utilisé suivant ces deux propriétés. La question de savoir si la prohibition concerne l'application sur le corps ou sur les vêtements est donc discutée.

Transcendantales, les odeurs permettent d'accéder au divin et de définir l'espace du sacré (muqaddas). Si leur rôle théorique est bien défini, leurs usages pratiques sont plus difficiles à étudier par manque de sources explicites. Certaines traces de cette fonction rituelle apparaissent néanmoins. Les fumigations sont ainsi fréquentes chez les soufis, contribuant à ouvrir les portes du monde spirituel (J.-Ch. Ducène). Ce rôle explique également l'usage de produits odorants dans le traitement des défunts. Concernant le safran, il est difficile de déterminer si sa prohibition pour les hommes, déjà soumise à la discussion des exégètes, était appliquée dans la vie quotidienne.

En effet, le champ des pratiques est vaste et hétérogène. Ce qui vaut pour l'élite ne vaut pas pour le peuple, les pratiques urbaines diffèrent des pratiques rurales, les faits divergent du droit et les textes ne reflètent pas nécessairement la praxis. Ainsi, l'élite respectaitelle réellement l'interdiction du safran ? Les discussions exégétiques touchaient-elles les comportements ou demeuraient-elles des exercices de réflexion théorique? Les écrits littéraires décrivant les mœurs des raffinés sont-ils plus réalistes que les textes normatifs? Le cas du safran est un bon exemple de ces questionnements. Les articles de M. Balda et A. Shinikov semblent en effet entrer en contradiction puisque la première compte le safran au nombre des parfums licites pour les hommes raffinés tandis que le second discute sa

25. SPERBER 1974, p. 129. À ce propos, voir BONNÉRIC à paraître et, dans ce volume, les articles de J. Bonnéric, J. Candau et A. Shinikov. 
prohibition justement pour les hommes. En réalité, tandis que M. Balda a examiné le choix des parfums, suivant le genre et la catégorie sociale, à partir de la littérature, A. Shinikov s'est interrogé sur le cadre de l'usage du safran d'un point de vue théorique. Il examine la prohibition du safran pour les hommes, posée par les hadiths, mais dont l'interprétation est commentée par les exégètes. La pratique ne suivait pas nécessairement les règles normatives. Par ailleurs, si M. Balda a choisi de traduire 'abìr par safran, il est possible que ce terme ait désigné un parfum particulier, dont il est difficile d'affirmer qu'il contenait du safran ${ }^{26}$. Dans ce cas, les pratiques des raffinés décrites dans le Kitäb al-muhibb ne seraient pas en contradiction avec la prohibition prophétique étudiée par A. Shinikov.

Il n'en demeure pas moins que les pratiques et comportements sont difficiles à restituer, en particulier lorsqu'ils sont populaires. Le cas de l'embaumement des mosquées, traité dans le dernier article de ce dossier thématique, est un bon exemple de ces difficultés. Les informations sur l'absence de produits odorants dans les mosquées sont en effet peu nombreuses et peu explicites, cette absence contrastant avec les cultes antérieurs et contemporains de l'islam. Dans cet article, différentes explications sont passées en revue. Ainsi, l'explication de cette absence par une faible ritualisation du culte dans les mosquées est invalidée. En revanche, une volonté de différenciation est une interprétation intéressante : la neutralité olfactive des mosquées pourrait trouver son origine dans la volonté de distinguer ces édifices, tant des bâtiments profanes (habitat, palais, etc.) que des autres édifices religieux (églises, synagogues, etc.) rendus sur-odorants par l'abondance de parfums qui y sont brûlés ou appliqués. Une interdiction énoncée par Ibn Taymiyya et citée par J.-Ch. Ducène dans son étude conforte à cette hypothèse puisque l'encens, comme produit magique et comme offrande, y est prohibé justement parce qu'il est courant dans les religions chrétienne et sabéenne. Pour ce juriste, l'encens n'est et ne doit être qu'un parfum.

\section{Conclusion}

Écrire l'histoire des odeurs n'est pas compulser l'histoire des odorants. Si ces derniers attirent plus souvent l'attention des chercheurs, c'est qu'ils ont une matérialité que n'ont pas l'odeur ou l'olfaction mais également parce que les sources sont plus loquaces à leur propos. J. Candau note bien dans son article que l'odeur est souvent assimilée à l'odorant, alors qu'elle en est la représentation cognitive. Or, d'un point de vue historique, l'odeur est plus riche car elle touche aux représentations culturelles, sociales et symboliques. Ce volume rend d'ailleurs bien compte de la fécondité et de la densité de ce thème. Odeurs et

26. En effet le safran simple est habituellement désigné par le terme za'farān. D’après le Kazimirski, ce terme désigne le safran ou un parfum composé de toutes sortes d'aromates. Adolf Wahrmund (1898) le traduit par les termes d'ambre, parfum ou safran. Il apparaît comme un parfum dans une notice de Joseph Héliodore Garcin de Tassy sur les fêtes populaires hindoues (GARCIN DE TASSY 1834, p. 229). En turc contemporain, il désigne, semble-t-il, un parfum fait d'ambre, de musc et de safran. Il est donc tout à fait possible que 'abìr ne désigne pas le safran, mais un parfum composé, contenant éventuellement du safran. 
odorants sont néanmoins indissociables : connaître les usages des odorants est par exemple nécessaire à l'examen des fonctions discriminatoires de l'odeur.

L'importance de l'odorat en Islam est difficile à évaluer et d'autres études seront nécessaires. Les notes olfactives des substances odorantes sont peu décrites par les auteurs médiévaux. Les odeurs sont principalement comparées à d'autres matières, odorantes le plus souvent. Ainsi, dans la poésie, le musc est comparé à l'odeur de la violette ou de l'orange amère. La note caractéristique de l'ambre, cette substance parfumée pourtant mentionnée par tant d'auteurs, n'est pas dépeinte comme chaude, boisée, animale, fleurie ou légèrement tabacée. Même son odeur désagréable, qui est en réalité celle qui précède une exposition longue à la lumière, est uniquement comparée à l'odeur du poisson. D'autre part, la couleur des substances odorantes est souvent mise en avant, par exemple comme topos littéraire, mais également pour reconnaître la qualité d'un produit comme l'ambre (Th. Buquet) ou le fumier (S. Chakhoum et H. Guenoune). Dans la poésie et la littérature, la couleur du musc ou de l'ambre fait également l'objet de descriptions, quasiment plus que l'odeur. La couleur et l'odeur de ces substances constituent un topos de comparaison avec la bien-aimée (son haleine ou son odeur pour la senteur, son apparence physique pour la couleur) et avec le vin. Néanmoins, lorsqu'Abū al-Qāsim déploie ses talents d'orateur pour injurier et outrager les notables d'Ispahan, l'odorat est le sens dans lequel il puise ses insultes favorites. La vue l'inspire également, tandis que l'ouïe et le goût paraissent marginalisés. M. Balda voit ici une différence avec la littérature occidentale où la laideur, plus que la pestilence, serait le signe de la perversion. A. Shinikov montre bien quant à lui qu'il est difficile d'établir une hiérarchie des sens dans le cas du rôle culturel du safran. La couleur, comme l'odeur du safran, paraissent jouer un rôle dans cette prohibition, qu'elle soit théorique ou non, car toutes deux revêtent une signification. Dans tous les cas, tant la richesse du vocabulaire des odeurs en arabe (B. Paoli), que le lien entre odeurs et sacré en hébreux (J. Sibony) ou que l'inventivité des auteurs médiévaux dans les insultes liées à ce thème (M. Balda) sont révélateurs de la place centrale, si ce n'est de l'odorat, du moins des odeurs.

Tous les aspects relatifs aux odeurs ne sont pas présents dans ce volume. Manquent des thèmes aussi fructueux que le rôle des odeurs dans les pratiques funéraires et le culte des saints, la gestion des pestilences dans les villes, le rapport entre odeurs et sexualité ou le travail des arômes en cuisine. Les mauvaises odeurs sont par ailleurs moins détaillées dans ce volume car elles ne sont abordées que par trois articles (S. Chakhoum et H. Guenoune ; M. Balda; J.-Ch. Coulon). Bien que certains thèmes intéressants n'apparaissent pas dans ce dossier thématique, celui-ci dresse néanmoins un portrait assez complet des approches possibles et de leur portée historique. En effet, étudier le rapport des hommes, en Islam, aux fragrances et aux pestilences offre l'occasion d'évoquer leur imaginaire social et symbolique. De même que l'anthropologie a montré que les odeurs pouvaient être des « marqueurs de frontières sociales ${ }^{27}$ ", l'analyse de l'exégèse et de la poésie en témoigne

27. Pour une approche archéologique de ces marqueurs sociaux, voir, dans ce volume, la contribution de J. Candau. Voir également : CANDAU 2013, DULAU 1998, LORCY 2011, ROUBIN 1989, VAN BEEK 1992. 
également. Les odeurs sont en outre une clef d'analyse du rapport des musulmans à la mort, au divin et au sacré. S'intéresser à ce thème révèle enfin les lacunes de chaque spécialité et les domaines encore à explorer. Par exemple, la synonymie et la richesse du lexique arabe des odeurs interrogent la constitution des dictionnaires anciens par les lexicographes dont il est difficile de déterminer s'ils prenaient en compte tous les dialectes de l'Arabie. L'indifférence des chercheurs en archéologie islamique pour le contexte de découverte des objets et pour leur fonctionnalité apparaît également lorsque l'on s'intéresse aux odeurs.

\section{Bibliographie}

ÁLAM Hūšang 1990, « Camphor », in Encyclopaedia Iranica 4/7, p. 743-747.

AKASOY Anna et YOELI-TLALIN Romit 2007, « Along the musk routes. Exchanges between Tibet and the Islamic world », Asian medicine 3, p. 217-240.

ALBERT Jean-Pierre 1990, Odeurs de sainteté. La mythologie chrétienne des aromates, Paris, Éditions de l'École des Hautes Études en Sciences Sociales.

ASHBROOK HARVEY Susan 2006, Scenting salvation. Ancient Christianity and the olfactory imagination, Berkeley, University of California Press.

ASHTOR Eliyzhu 1968, Essai sur l'alimentation des diverses classes sociales dans l'Orient médiéval », Annales. Économies, Sociétes, Civilisations 23/5, p. 1017-1053.

AubAile-SAlLENAVE Françoise 1994, «Parfums, épices et condiments dans l'alimentation arabe médiévale », in Manuela MARÍN et David WAINES (éd.), La alimentación en las culturas islámicas, Madrid, Agencia Española de Cooperación Internacional, p. 217-249.

AUBAILE-SALLENAVE Françoise 1998, « Bân, un parfum et une image de la souplesse : l'histoire d'un arbre dans le monde arabo-musulman », Res orientales XI, dossier thématique : Parfums d'Orient, Ryka GYSELEN (éd.), p. 9-27.

AUBAILE-SALLENAVE Françoise 1999, « Le Souffle des parfums : un essai de classification des odeurs chez les Arabo-musulmans ", in Danielle MUSSET et Claudine FABRE-VASSAS (éd.), Odeurs et parfums, $121^{\mathrm{e}}$ congrès national des sociétés historiques et scientifiques, Nice, 1996, Paris, Éditions du CTHS p. 93-115.

AUBAILE-SALLENAVE Françoise 2004, «Corps, odeurs, parfums dans les sociétés arabo-musulmanes », in Jane COBBI et Robert DULAU, Sentir. Pour une anthropologie des odeurs, Paris, L'Harmattan, p. 181-192.

BALSAMO Isabelle (éd.) 2006, Odeurs, Paris, Maison des Sciences de l'Homme (Terrain 47).

BESSARD Fanny 2008, "Pratiques sanitaires, produits d'hygiène et de soin dans les bains médiévaux (VIII ${ }^{\mathrm{e}}-\mathrm{IX}^{\mathrm{e}}$ siècles) », Bulletin d'Études Orientales LVII, p. 111-125.

BOHAS Georges et SAGUER Abderrahim 2012, Le son et le sens. Fragment d'un dictionnaire étymologique de l'arabe classique, Beyrouth, Presses de l'Ifpo (PIFD 277).

BoISSON Claude 1997, «La dénomination des odeurs: variations et régularités linguistiques », Intellectica $1 / 24$, p. 29-49.

BONNÉRIC Julie 2012, « Les huiles et leurs usages en terre d'Islam à partir du VII ${ }^{\mathrm{e}}$ s. », in Dominique FRÈRE et Laurent HUGOT (éd.), Les huiles parfumées en Méditerranée occidentale et en Gaule (VIII av.-VIII aps. J.-C.), Rennes, Presses universitaires de Rennes, p. 307-314. 
BONNÉRIC Julie à paraître, «L'odeur du guerrier : un parfum d'immortalité sur le champ de bataille », in Mathieu EYCHENNE, Stéphane PRADINES et Abbès ZOUACHE (éd.), Guerre et paix dans le Proche-Orient médiéval. Histoire. Archéologie. Anthropologie, Le Caire, Ifao/Ifpo.

CANDAU Joël 2013, «Une théorie sensorielle de l'identité », in Paul-Louis CoLon (éd.), Ethnographier les sens, Paris, Éditions Petra, p. 201-234.

CASEAU Béatrice 2013, «L'encens au $7^{\mathrm{e}}$ et $8^{\mathrm{e}}$ siècle : un marqueur du commerce en Méditerranée? ", in Apóstolos F. KRALÍDĪs (éd.), Byzantium and the Arab World: Encounter of civilizations: proceedings of the international symposium (Thessaloniki, 16-18 december 2011), Thessalonique, Aristotéleio Panapistîmio, p. 105-116.

CLASSEN Constance 1993, Worlds of Sense, Londres, Routledge.

CLASSEN Constance, HoweS David et SyNnOTT Anthony 1994, The Cultural History of Smell, LondresNew York, Routledge.

CORBIN Alain 1982, Le miasme et la jonquille, Paris, Aubier-Montaigne (Collection historique).

DE GOEJE Jan 1907, « L'encensement des morts chez les anciens Arabes », in Actes du XIV ${ }^{e}$ congrès international des orientalistes, Alger, 1905, Paris, Leroux, III, p. 3-7.

DETIENNE Marcel 2007, Les jardins d'Adonis, Paris, Gallimard (Folio histoire 149).

DIETRICH Albert 1983, «Lubān », in Encyclopédie de l'Islam (2éd.), Leyde, E.J. Brill, V, p. 792-793.

DIETRICH Albert 1993, « Misk », in The Encyclopaedia of Islam, Leyde-New York, E.J. Brill, VII, p. 142-143.

DiETRICH Albert, 1997, « Kāfūr », in The Encyclopaedia of Islam, Leyde, E.J. Brill, IV, p. 417-418.

DULAU Robert 1998, «Exploration du champ du senti à Pondichéry », in Robert DULAU et Jean-Robert PITTE (éd.), Géographie des odeurs, Paris, L'Harmattan, p. 81-118.

EVANS Suzanne 2002, "The scent of a martyr », Numen 49/2, p. 193-211.

EVERSHED Richard, VAN BERGEN Pim, PEAKMAN Torren, LEIGH-FIRBANK Elizabeth, HoRTON Mark, EDWARDS David, BidDle Martin, KJøLBYE-BIDdLe Birthe et RoWleY-ConWY Peter 1997, «Archaeological frankincense », Nature 390, p. 667-778.

FISCHEL Walter J. 1958, «The Spice Trade in Mamluk Egypt: A Contribution to the Economic History of Medieval Islam ", Journal of the Economic and Social History of the Orient 1/2, p. 157-174.

GARCÍA-SÁNCHEZ Expiración 1997, "La consommation des épices et des plantes aromatiques en al-Andalus », Médiévales 16/33, p. 41-53.

GARCÍA-SÁNCHEZ Expiración 1998, « Les techniques de distillation de l'eau de rose » in Rika GYSELEN (éd.), Res orientales XI, dossier thématique : Parfums d'Orient, Ryka GYSELEN (éd.), p. 125-139.

GARCIN DE TASSY Joseph Héliodore 1834, « Notice sur les fêtes populaires des Hindous, d'après les ouvrages hindoustani », Nouveau Journal asiatique XIII, p. 219-239.

GELL Alfred 2006, «Parfum, symbolisme et enchantement », Terrain 47, p. 19-34.

GIGANDET Suzanne 2002, Al-Risāla al-Hārūniyya de Masīh b. Hakam al-Dimašqū, médecin, Damas, Institut français d'études arabes de Damas.

KAZIMIRSKI Albert DE BIBERSTEIN- 1860, Dictionnaire arabe-français, Paris, Maisonneuve et Cie, 2 vol.

HIRSCH Hadas 2013, "Cosmetics and gender: Perfumes in medieval legal Muslim sources », H\&PC Today 8/1, p. 13-17. [En ligne] http://www.teknoscienze.com/Articles/HPC-Today-Cosmetics-andgender-Perfumes-in-medieval-legal-Muslim-sources.aspx\#.VCryuNXLfRY

HOWES David 1986, "Le sens sans parole: vers une anthropologie de l'odorat», Anthropologie et Sociétés 10/3, p. 29-45. 
HOWES David 2003, Sensual Relations. Engaging the Senses in Culture and Social Theory, Ann Arbor, University of Michigan Press.

KING Anya H. 2008, "The Importance of Imported Aromatics in Arabic Culture: Illustrations from Pre-Islamic and Early Islamic Poetry », Journal of Near Eastern Studies 67/3, p. 175-189.

KING Anya H. 2007, The Musk Trade and the Near East in the Early Medieval Period, PhD Dissertation, Bloomington, Indiana University (Advisor: Christopher I. Beckwith), Ann Harbor, ProQuest, 2008.

LARDELLIER Pascal (éd.) 2003, À fleur de peau : corps, odeurs, parfums, Paris, Belin.

LE GUÉRER Annick 2003, «L'odorat, un sixième sens? », in Pascal LARDELLIER (éd.), À fleur de peau : corps, odeurs, parfums, Paris, Belin, p. 15-20.

LE GUÉRER Annick 2005, Le Parfum des origines à nos jours, Paris, Odile Jacob.

LE MAGUER Sterenn 2011, « Typology of incense-burners of the Islamic period », Proceedings of the Seminar for Arabian Studies 41, p. 173-195.

LEV Efraim et ZOHAR Amar 2013, «Trends in the use of perfumes and incense in the Near East after the Muslim conquests ", Journal of the Royal Asiatic Society 3/23, p. 11-30.

LEVEY Martin 1961, «Ibn Māsawayh and his Treatise on Simple Aromatic Substances. Studies in the History of Arabic Pharmacology, I ", Journal of the History of Medicine and Allied Science 16/4, p. 394-410.

LORCY Armelle 2011, « Le Puant, le mariné et le “civilisé” », Techniques \& Culture 57, p. 227-237.

MARIN Manuela 1998, «The perfumed kitchen: Arab Cookbooks from the Islamic East, »,Res orientales XI, dossier thématique : Parfums d'Orient, Ryka GYSELEN (éd.), p. 159-166.

MCHugh James 2012, Sandalwood and Carrion: Smell in Indian Religion and Culture, New York, Oxford University Press.

Мicнот Jean 1978, « L'épitre d'Avicenne sur le parfum », Bulletin de philosophie médiévale 20, p. 53-57.

MILWRIGHT Marcus 2003, « The balsam of Matariyya: An exploration of a Medieval panacea », Bulletin of the School of Oriental and African Studies 66/2, p. 193-209.

MOTTAHEDIN Žāl 1985, « 'Anbar », in Encyclopaedia Iranica 2, p. 4-5.

NASRALLAH Nawal 2007, Annals of the Caliphs' Kitchens: Ibn Sayyār al-Warrāq's Tenth-Century Baghdadi Cookbook, Leyde-Boston, E.J. Brill (Islamic History and Civilization 70), 2007.

RouBIN Lucienne A. 1989, Le monde des odeurs, Paris, Méridiens Klincksieck.

RUSKA Julius et PLESSNER Martin 1986, « 'Anbar », in Encyclopaedia of Islam. New Edition, Leyde, E.J. Brill, I, p. 484.

SANAGUSTIN Floréal 1998, "Parfums et pharmacologie en Orient médiéval : savoirs et représentations », Res orientales XI, dossier thématique : Parfums d'Orient, Ryka GYSELEN (éd.), p. 189-202.

SPERBER Dan 1974, Le Symbolisme en général, Paris, Herman.

STÉPHAN Noucha 1998, « Le camphre dans les sources arabes et persanes. Production et usages », in Claude Guillot (éd.), Histoire de Barus, Sumatra. Le site de Lobu Tua, vol. 1, Études et documents, Paris, Cahiers de l'Archipel 30, p. 225-241.

TRITTON Arthur Stanley 1937, «Muslim Funeral Customs », Bulletin of the School of Oriental Studies IX, p. 653-661. 
VAN BEEK Walter E.A. 1992, « The Dirty Smith: Smell as a Social Frontier among the Kapsiki/Higi of North Cameroon and North-Eastern Nigeria ", Africa 62/1, p. 38-58.

WAHRMUND Adolf 1898, Handwörterbuch der neu-arabischen und deutschen Sprache, Giessen, J. Rickersche Verlags-Buchhandlung, 2 vol.

WAINES David et SANAGUSTIN Floréal 2002, «Za'farān », in The Encyclopaedia of Islam, Leyde, E.J. Brill, XI, p. 381-382. 